\title{
On generalized $A$ - difference strongly summable sequence spaces defined by ideal convergence on a real $n$-normed space
}

Ekrem Savas

Correspondence: ekremsavas@yahoo.com Department of Mathematics, Istanbul Commerce University, Uskudar-Istanbul, Turkey

\begin{abstract}
In this article, we shall introduce a new class of ideal convergent (briefly 1 convergent) sequence spaces using, infinite matrix, an Orlicz function and difference operator defined on $n$-normed spaces. We study these spaces for some linear topological structures and algebraic properties. We also give some relations related to these sequence spaces.

Mathematics Subject Classification 2010: 40A05; 40B50; 46A19; 46A45.

Keywords: I-convergence, infinite matrix, difference space, $n$-norm, Orlicz function
\end{abstract}

\section{Introduction}

The idea of statistical convergence was given by Zygmund [1] in the first edition of his monograph published in Warsaw in 1935. The concept of statistical convergence was introduced by Fast [2] and Schoenberg [3]. Over the years and under different names statistical convergence was discussed in the theory of Fourier analysis, ergodic theory, number theory, measure theory, turnpike theory and Banach spaces. Later on it was further investigated from sequence point of view and linked with the summability theory by Fridy [4] and many others. The idea is based on the notion of natural density of subsets of $\mathbf{N}$, the set of positive integers, which is defined as follows: The natural density of a subset $E$ of natural numbers is denoted by $\delta(E)$ and is defined by

$$
\delta(E)=\lim _{n \rightarrow \infty} \frac{1}{n}|\{k \in E: k \leq n\}|,
$$

where the vertical bar denotes the cardinality of the enclosed set.

Kastyrko et al. [5] introduced the concept of $I$-convergence of sequences in a metric space and studied some properties of such convergence. Since then many researchers have studied these subjects and obtained various results (see [6-9]). Note that $I$-convergence is an interesting generalization of statistical convergence.

The notion of difference sequence space was introduced by Kizmaz [10]. It was further generalized by Et and Colak [11] by introducing the sequence spaces $\ell_{\infty}\left(\Delta^{s}\right), c$ $\left(\Delta^{s}\right), c_{0}\left(\Delta^{s}\right)$. For a non negative integer $s$, the generalized difference sequence spaces are defined as follows: For a given sequence space $X$ we have

$$
X\left(\Delta^{s}\right)=\left\{x=\left(x_{k}\right) \in w:\left(\Delta^{s} x_{k}\right) \in X\right\},
$$

(c) 2012 Savas; licensee Springer. This is an Open Access article distributed under the terms of the Creative Commons Attribution License (http://creativecommons.org/licenses/by/2.0), which permits unrestricted use, distribution, and reproduction in any medium, provided the original work is properly cited. 
where $\Delta^{s} x_{k}=\Delta^{s-1} x_{k}-\Delta^{s-1} x_{k+1}, \Delta^{0} x_{k}=x_{k}$, for all $k \in \mathbf{N}$, the difference operator is equivalent to the following binomial representation:

$$
\Delta^{s} x_{k}=\sum_{v=0}^{s}(-1)^{v}\left(\begin{array}{l}
s \\
v
\end{array}\right) x_{k+v}
$$

Taking $s=1$, we get the spaces $\ell_{\infty}(\Delta), c(\Delta), c_{0}(\Delta)$, introduced and studied by Kizmaz [10].

The concept of 2-normed space was initially introduced by Gahler [12], in the mid of 1960 's, while that of $n$-normed spaces can be found in Misiak [13]. Since then, many others have studied this concept and obtained various results, (see Gunawan [14], Gunawan and Mashadi $[15,16])$. The notion of ideal-convergence in 2-normed spaces was introduced and studied in $[17,18]$ and [19]. Later on it was extended to $n$-normed spaces by Gurdal and Sahiner [20], Hazarika [21] and Savas [22].

Let $X$ be a non-empty set, then a family of sets $I \subset 2^{X}$ (the class of all subsets of $X$ ) is called an ideal if and only if for each $A, B \in I$, we have $A \cup B \in I$ and for each $A \in$ $I$ and each $B \subset A$, we have $B \in I$. A non-empty family of sets $F \subset 2^{X}$ is a filter on $\mathrm{X}$ if and only if $\Phi \notin F$, for each $A, B \in F$, we have $A \cap B \in F$ and each $A \in F$ and each $A$ $\subset B$, we have $B \in F$. An ideal $I$ is called non-trivial ideal if $I \neq \varnothing$ and $X \notin I$. Evidently $I$ $\subset 2^{X}$ is a non-trivial ideal if and only if $F=F(I)=\{X-A: A \in I\}$ is a filter on $X$. A non-trivial ideal $I \subset 2^{X}$ is called admissible if and only if $\{\{x\}: x \in X\} \subset I$. A non-trivial ideal $I$ is maximal if there cannot exists any non-trivial ideal $J \neq I$ containing $I$ as a subset. Further details on ideals of $2^{X}$ can be found in Kostyrko et. al [5].

An Orlicz function is a function $M:[0, \infty) \rightarrow[0, \infty)$, which is continuous, nondecreasing and convex with $M(0)=0, M(x)>0$, as $x>0$ and $M(x) \rightarrow \infty$, as $x \rightarrow \infty$ (see [23]).

An Orlicz function $M$ is said to satisfy $\Delta_{2}$ - condition for all values of $u$, if there exists constant $K>0$ such that $M(2 u) \leq K M(u), u \geq 0$.

Lindenstrauss and Tzafriri [24] studied some Orlicz type sequence spaces defined as follows:

$$
\ell_{M}=\left\{\left(x_{k}\right) \in w: \sum_{k=1}^{\infty} M\left(\frac{\left|x_{k}\right|}{\rho}\right)<\infty, \text { for some } \rho>0\right\} .
$$

The space $\ell_{M}$ with the norm

$$
\|x\|=\inf \left\{\rho>0: \sum_{k=1}^{\infty} M\left(\frac{\left|x_{k}\right|}{\rho}\right) \leq 1\right\}
$$

becomes a Banach space which is called an Orlicz sequence space. The space $\ell_{M}$ is closely related to the space $\ell_{p}$ which is an Orlicz sequence space with $M(t)=|t|^{p}$, for 1 $\leq p<\infty$. Subsequently Orlicz function was used to define sequence spaces by Parashar and Choudhary [25] and many others (see, [26-29]).

The following well-known inequality will be used throughout the article. Let $p=\left(p_{k}\right)$ be any sequence of positive real numbers with $0 \leq p_{k} \leq \sup _{k} p_{k}=G, D=\max \left\{1,2^{G-1}\right\}$ then

$$
\left|a_{k}+b_{k}\right|^{p_{k}} \leq D\left(\left|a_{k}\right|^{p_{k}}+\left|b_{k}\right|^{p_{k}}\right)
$$


for all $k \in N$ and $a_{k}, b_{k} \in C$. Also $|a|^{p_{k}} \leq \max \left\{1,|a|^{G}\right\}$ for all $a \in C$.

In 2001, Gunawan and Mashadi in [15] gave the following definitions.

\section{Definitions and preliminaries}

Let $n$ bea non-negative integer and $X$ be a real vector space of dimension $d \geq n$ ( $d$ may be infinite). A real-valued function $\| ., . .$, . $\|$ on $X^{n}$ satisfying the following conditions:

(1) $\left\|\left(x_{1}, x_{2}, \ldots, x_{n}\right)\right\|=0$ if and only if $x_{1}, x_{2}, \ldots, x_{n}$ are linearly dependent,

(2) $\left\|\left(x_{1}, x_{2}, \ldots, x_{n}\right)\right\|$ is invariant under permutation,

(3) $\left\|\alpha\left(x_{1}, x_{2}, \ldots, x_{n}\right)\right\|=|\alpha|\left\|\left(x_{1}, x_{2}, \ldots, x_{n}\right)\right\|$, for any $\alpha \in \mathbf{R}$,

(4) $\left\|\left(x+\bar{x}, x_{2}, \ldots, x_{n}\right)\right\| \leq\left\|\left(x_{1}, x_{2}, \ldots, x_{n}\right)\right\|+\left\|\left(\bar{x}, x_{2}, \ldots, x_{n}\right)\right\|$

is called an $n$-norm on $X$ and the pair $(X,\|., \ldots,\|$.$) is called an n$-normed space.

A trivial example of an $n$-normed space is $X=\mathbf{R}^{n}$, equipped with the Euclidean $n$ norm $\left\|\left(x_{1}, x_{2}, \ldots, x_{n}\right)\right\|_{E}=$ the volume of the $n$-dimensional parallelpiped spanned by the vectors $x_{1}, x_{2}, \ldots, x_{n}$ which may be given explicitly by the formula

$$
\left\|\left(x_{1}, x_{2}, \ldots, x_{n}\right)\right\|_{E}=\left|\operatorname{det}\left(x_{i j}\right)\right|=\operatorname{abs}\left(\operatorname{det}\left(<x_{i}, x_{j}>\right)\right)
$$

where $x_{i}=\left(x_{i 1}, x_{i 2}, \ldots, x_{i n}\right) \in \mathbf{R}^{n}$ for each $i=1,2,3 \ldots, n$.

Let $(X,\|., \ldots,\|$.$) be an n$-normed space of dimension $d \geq n \geq 2$ and $\left\{a_{1}, a_{2}, \ldots, a_{n}\right\}$ be a linearly independent set in $X$. Then the function $\|., . . .,\|_{\infty}$ on $X^{n-1}$ is defined by

$$
\left\|\left(x_{1}, x_{2}, \ldots, x_{n}\right)\right\|_{\infty}=\max _{1 \leq i \leq n}\left\{\left\|x_{1}, x_{2}, \ldots, x_{n-1}, a_{i}\right\|\right\}
$$

defines as $(n-1)$-norm on $X$ with respect to $\left\{a_{1}, a_{2}, \ldots a_{n}\right\}$ and this is known as the derived $(n-1)$-norm (see [14]).

The standard $n$-norm on $X$ a real inner product space of dimension $d \geq n$ is as follows:

$$
\left\|\left(x_{1}, x_{2}, \ldots, x_{n}\right)\right\| s=\left[\operatorname{det}\left(<x_{i}, x_{j}>\right)\right]^{\frac{1}{2}},
$$

where $<$, > denotes the inner product on $X$. If we take $X=\boldsymbol{R}^{n}$ then this $n$-norm is exactly the same as the Euclidean $n$-norm $\left\|\left(x_{1}, x_{2}, \ldots, x_{n}\right)\right\|_{E}$ mentioned earlier. For $n=$ 1 this $n$-norm is the usual norm $\left\|x_{1}\right\|=\sqrt{\left.<x_{1}, x_{1}\right\rangle}$ (for further details see Gunawan [14]).

We first introduce the following definitions (see also [21]).

Definition 2.1. A sequence $\left(x_{k}\right)$ in an $n$-normed space $(X,\|., \ldots,\|$.$) is said to be$ convergent to some $L \in X$ with respect to the $n$-norm if for each $\varepsilon>0$ there exists an positive integer $n_{0}$ such that $\left\|x_{k}-L, z_{1}, z_{2}, \ldots, z_{n-1}\right\|<\varepsilon$, for all $k \geq n_{0}$ and for every $z_{1}$, $z_{2}, \ldots, z_{n-1} \in X$.

Definition 2.2. A sequence $\left(x_{k}\right)$ in an $n$-normed space $(X,\|., ., \ldots,\|$.$) is said to be I$ convergent to some $L \in X$ with respect to the $n$-norm if for each $\varepsilon>0$ such that the set $\left\{k \in \mathbf{N}:\left\|x_{k}-L, z_{1}, z_{2}, \ldots, z_{n-1}\right\| \geq \varepsilon\right\}$ belongs to $I$, for every $z_{1}, z_{2}, \ldots, z_{n-1} \in X$.

Definition 2.3. A sequence $\left(x_{k}\right)$ in a normed space $(X,\|\|$.$) is said to be I-bounded if$ there exists an positive integer $M>0$ such that the set $\left\{k \in \mathbf{N}:\left\|x_{k}, z_{1}, z_{2}, \ldots, z_{n-1}\right\| \geq\right.$ $M$ ) belongs to $I$, for every $z_{1}, z_{2}, \ldots, z_{n-1} \in X$. 
Definition 2.4. [6]. A sequence space $E$ is said to be solid (or normal) if $\left(\alpha_{k} x_{k}\right) \in E$, whenever $\left(x_{k}\right) \in E$ and for all sequence $\left(\alpha_{k}\right)$ of scalars with $\left|\alpha_{k}\right| \leq 1$, for all $k \in \mathbf{N}$.

Let $K=\left\{k_{1}<k_{2}<\cdots\right\} \subseteq \mathbf{N}$ and $E$ be a sequence space. A $K$-step space of $E$ is a sequence space $\lambda_{K}^{E}=\left\{\left(x_{k_{n}}\right) \in w:\left(x_{k}\right) \in E\left(k_{n}\right) \in K\right\}$.

$A$ canonical preimage of a sequence $\left\{\left(x_{k_{n}}\right)\right\} \in \lambda_{K}^{E}$ is a sequence $\left\{y_{n}\right\} \in w$ defined as

$$
y_{n}= \begin{cases}x_{n}, & \text { if } n \in K \\ 0, & \text { otherwise }\end{cases}
$$

A canonical preimage of a step space $\lambda_{K}^{E}$ is a set of canonical preimages of all elements in $\lambda_{K}^{E}$ is in canonical preimage of $\lambda_{K}^{E}$ if and only if $y$ is canonical preimage of some $x \in E$.

Definition 2.5. A sequence space $E$ is said to be monotone if it contains the canonical preimages of its step spaces.

1) If we take $I=I_{f}=\{A \subseteq \mathbf{N}: A$ is a finite subset $\}$. Then $I_{f}$ is a non-trivial admissible ideal of $\mathbf{N}$ and the corresponding convergence coincide with the usual convergence.

2) If we take $I=I_{\delta}=\{A \subseteq \mathbf{N}: \delta(A)=0\}$ where $\delta(A)$ denote the asymptotic density of the set $A$. Then $I_{\delta}$ is a non-trivial admissible ideal of $\mathbf{N}$ and the corresponding convergence coincide with the statistical convergence.

Lemma 2.1. ([30]). Every normal space is monotone.

Lemma 2.2. [15]. Every n-normed space is an (n-r)-normed space for all $r=1,2, \ldots$, $n-$

1. In particular every n-normed space is a normed space.

In this article, we study some new ideal convergent sequence spaces on $n$-normed spaces by using Orlicz functions, infinite matrix and generalized difference operator.

\section{Main results}

Before we state our main results, first we shall present some new ideal convergent sequence spaces by combining an infinite matrix and Orlicz function and study their linear topological structures. Also we give some relations related to these sequence spaces.

Let $I$ be an admissible ideal of $\mathbf{N}$, and let $p=\left(p_{k}\right)$ be a bounded sequence of positive real numbers for all $k \in \mathbf{N}$ and $A=\left(a_{n k}\right)$ an infinite matrix. Let $M$ be an Orlicz function and $(X,\|. . ., \ldots,\|$.$) be an n$-normed space. Further $w(n-X)$ denotes the spaces of all $X$-valued sequence spaces. For every $z_{1}, z_{2}, \ldots, z_{n-1} \in X$, for each $\varepsilon>0$ and for some $\rho>0$ we define the following sequence spaces:

$$
\begin{aligned}
& w^{I}\left[A, \Delta^{s}, M, p,\|., \ldots ., .\|\right]=\left\{x=\left(x_{k}\right) \in w(n-X): \text { for a given } \varepsilon>0,\{n \in \mathbf{N}:\}\right\} \\
& \left.\sum_{k=1}^{\infty} a_{n k}\left[M\left(\left\|\frac{\Delta^{s} x_{k-L}}{\rho}, z_{1}, z_{2}, \ldots, z_{n-1}\right\|\right)\right]^{p_{k}} \geq \varepsilon \in I, \text { for } L \in X\right\},
\end{aligned}
$$




$$
\begin{aligned}
& w_{0}^{I}\left[A, \Delta^{s}, M, p,\|., \ldots . .,\|\right]= \\
& \left\{x=\left(x_{k}\right) \in w(n-X): \text { for a given } \varepsilon>0,\left\{n \in \mathrm{N}: \sum_{k=1}^{\infty} a_{n k}\left[M\left(\left\|\frac{\Delta^{s} x_{k}}{\rho}, z_{1}, z_{2}, \ldots, z_{n-1}\right\|\right)\right]^{p_{k}} \geq \varepsilon\right\} \in I\right\}, \\
& w_{\infty}^{I}\left[A, \Delta^{s}, M, p\|., \ldots . .,\|\right]= \\
& \left\{x=\left(x_{k}\right) \in w(n-X): \exists K>0 \text { s.t. }\left\{n \in \mathrm{N}: \sum_{k=1}^{\infty} a_{n k}\left[M\left(\left\|\frac{\Delta^{s} x_{k}}{\rho}, z_{1}, z_{2}, \ldots, z_{n-1}\right\|\right)\right]^{p_{k}} \geq K\right\} \in I\right\},
\end{aligned}
$$

Let us consider a few special cases of the above sets.

(i) If $s=0$ then we obtain the spaces as $w^{I}[A, M, p,\|., \ldots . . .\|$,$] ,$ $w_{\infty}^{I}[A, M, p,\|., \ldots . .\|],. w_{\infty}^{I}[A, M, p,\|., \ldots . .\|$.$] , and w_{\infty}[A, M, p,\|., \ldots . . .\|$.$] , from$ the above sequence spaces.

(ii) If $s=1$, then above spaces are denoted by $w^{I}[A, \Delta M, p,\|., \ldots . . .\|$,$] ,$ $w_{0}^{I}[A, \Delta, M, p,\|., \ldots . .\|$.$] , and w_{\infty}^{I}[A, \Delta, M, p,\|., \ldots .\|$.$] .$

(iii) If $M(x)=x$ for all $x \in[0, \infty)$ then we obtain the spaces by $w^{I}\left[A, \Delta^{s}, p, \| ., \ldots .\right.$. , $. \|], w_{0}^{I}\left[A, \Delta^{s}, p,\|., \ldots .\|.\right]$, and $w_{\infty}^{I}\left[A, \Delta^{s}, p,\|., \ldots .\|.\right]$ from the above sequence spaces.

(iv) If $p=\left(p_{k}\right)=(1,1,1 \ldots)$, then above spaces becomes $w^{I}\left[A, \Delta^{s}, M,\|., \ldots . . .\|,\right]$, $w_{0}^{I}\left[A, \Delta^{s}, M,\|., \ldots .\|.\right]$, and $w_{\infty}^{I}\left[A, \Delta^{s}, M,\|., \ldots . .\|.\right]$.

(v) If we take $A=(C, 1)$, i.e., the Cesàro matrix, then the above classes of sequences are denoted by $w^{I}\left[\Delta^{s}, M, p,\|., \ldots . . .\|.\right], w_{0}^{I}\left[\Delta^{s}, M, p,\|., \ldots .\|.\right]$, and $w_{\infty}^{I}\left[\Delta^{s}, M, p,\|., \ldots . .\|.\right]$.

(vi) If we take $A=\left(a_{n k}\right)$ is a de la Valée Poussin mean, i.e.,

$$
a_{n k}=\left\{\begin{array}{lc}
\frac{1}{\lambda_{n}}, \text { if } k \in I_{n}=\left[n-\lambda_{n}+1, n\right], \\
0, \quad \text { otherwise }
\end{array}\right.
$$

where $\left(\lambda_{n}\right)$ is a non-decreasing sequence of positive numbers tending to $\infty$ and $\lambda_{n+1}$ $\leq \lambda_{n}+1, \lambda_{1}=1$, then the above spaces are denoted by $w^{I}\left[\lambda, \Delta^{s}, p,\|., \ldots . . .\|.\right]$, $w_{0}^{I}\left[\lambda, \Delta^{s}, p,\|., \ldots .\|.\right]$, and $w_{\infty}^{I}\left[\lambda, \Delta^{s}, p,\|., \ldots .\|.\right]$.

(vii) By a lacunary $\theta=\left(k_{r}\right) ; r=0,1,2, \ldots$ where $k_{0}=0$, we shall mean an increasing sequence of non-negative integers with $k_{r}-k_{r-1} \rightarrow \infty$ as $r \rightarrow \infty$. The intervals determined by $\theta$ will be denoted by $I_{r}=\left(k_{r-1}, k_{r}\right]$ and $h_{r}=k_{r}-k_{r-1}$.

As a final illustration let

$$
a_{n k}=\left\{\begin{array}{c}
\frac{1}{h_{r}}, \text { if } k_{r-1}<k \leq k_{r} \\
0, \quad \text { otherwise }
\end{array}\right.
$$

Then we have the above classes of sequences by $w^{I}\left[\theta, \Delta^{s}, p,\|., \ldots . . .\|.\right]$, $w_{0}^{I}\left[\theta, \Delta^{s}, p,\|., \ldots .\|.\right]$, and $w_{\infty}^{I}\left[\theta, \Delta^{s}, p,\|., \ldots .\|.\right]$.

Theorem 3.2. $w^{I}\left[A, \Delta^{s}, M, p,\|., \ldots . . .,\|.\right], w_{0}^{I}\left[A, \Delta^{s}, M, p,\|. \ldots . .\|.\right]$ and $w_{\infty}^{I}\left[A, \Delta^{s}, M, p,\|., \ldots . .\|.\right]$, are linear spaces. 
Proof. We shall prove the theorem for the space $w_{0}^{I}\left[A, \Delta^{s}, M, p,\|., \ldots . .\|,\right]$ only and the others can be proved by the same way. Let $x=\left(x_{k}\right)$ and $y=\left(y_{k}\right)$ be two elements in $w_{0}^{I}\left[A, \Delta^{s}, M, p,\|., \ldots . .\|.\right]$. Then there exist $\rho_{1}>0$ and $\rho_{2}>0$ and for every $z_{1}, z_{2}, \ldots$, $z_{n-1} \in X$ such that

$$
A_{\frac{\varepsilon}{2}}=\left\{n \in \mathbf{N}: \sum_{k=1}^{\infty} a_{n k}\left[M\left(\left\|\frac{\Delta^{s} x_{k}}{\rho_{1}}, z_{1}, z_{2}, \ldots, z_{n-1}\right\|\right)\right]^{p_{k}} \geq \frac{\varepsilon}{2}\right\} \in I
$$

and

$$
B_{\frac{\varepsilon}{2}}=\left\{n \in \mathbf{N}: \sum_{k=1}^{\infty} a_{n k}\left[M\left(\left\|\frac{\Delta^{s} y_{k}}{\rho_{2}}, z_{1}, z_{2}, \ldots, z_{n-1}\right\|\right)\right]^{p_{k}} \geq \frac{\varepsilon}{2}\right\} \in I
$$

Let $\alpha, \beta$ be two scalars in R. Since $\|., ., \ldots,$.$\| is an n$-norm, $\Delta^{s}$ is linear and the continuity of the Orlicz function $M$, the following inequality holds:

$$
\begin{gathered}
\sum_{k=1}^{\infty} a_{n k}\left[M\left(\left\|\frac{\Delta^{s}\left(\alpha x_{k}+\beta y_{k}\right)}{|\alpha| \rho_{1}+|\beta| \rho_{2}}, z_{1}, z_{2}, \ldots, z_{n-1}\right\|\right)\right]^{p_{k}} \\
\leq D \sum_{k=1}^{\infty} a_{n k}\left[\frac{|\alpha|}{|\alpha| \rho_{1}+|\beta| \rho_{2}} M\left(\left\|\frac{\Delta^{s} x_{k}}{\rho_{1}}, z_{1}, z_{2}, \ldots, z_{n-1}\right\|\right)\right]^{p_{k}} \\
+D \sum_{k=1}^{\infty} a_{n k}\left[\frac{|\beta|}{|\alpha| \rho_{1}+|\beta| \rho_{2}} M\left(\left\|\frac{\Delta^{s} y_{k}}{\rho_{2}}, z_{1}, z_{2}, \ldots, z_{n-1}\right\|\right)\right]^{p_{k}} \\
\leq D K \sum_{k=1}^{\infty} a_{n k}\left[M\left(\left\|\frac{\Delta^{s} x_{k}}{\rho_{1}}, z_{1}, z_{2}, \ldots, z_{n-1}\right\|\right)\right]^{p_{k}}+D K \sum_{k=1}^{\infty} a_{n k}\left[M\left(\left\|\frac{\Delta^{s} y_{k}}{\rho_{2}}, z_{1}, z_{2}, \ldots, z_{n-1}\right\|\right)\right]^{p_{k}},
\end{gathered}
$$

where $K=\max \left\{1,\left(\frac{|\alpha|}{|\alpha| \rho_{1}+|\beta| \rho_{2}}\right),\left(\frac{|\beta|}{|\alpha| \rho_{1}+|\beta| \rho_{2}}\right)\right\}$

From the above relation we get

$$
\begin{gathered}
\left\{n \in \mathbf{N}: \sum_{k=1}^{\infty} a_{n k}\left[M\left(\left\|\frac{\Delta^{s}\left(\alpha x_{k}+\beta y_{k}\right)}{\left(|\alpha| \rho_{1}+|\beta| \rho_{2}\right)}, z_{1}, z_{2}, \ldots, z_{n-1}\right\|\right)\right]^{p_{k}} \geq \varepsilon\right\} \\
\subseteq\left\{n \in \mathbf{N}: D K \sum_{k=1}^{\infty} a_{n k}\left[M\left(\left\|\frac{\Delta^{s} x_{k}}{\rho_{1}}, z_{1}, z_{2}, \ldots, z_{n-1}\right\|\right)\right]^{p_{k}} \geq \frac{\varepsilon}{2}\right\} \\
\cup\left\{n \in \mathbf{N}: D K \sum_{k=1}^{\infty} a_{n k}\left[M\left(\left\|\frac{\Delta^{s} y_{k}}{\rho_{2}}, z_{1}, z_{2}, \ldots, z_{n-1}\right\|\right)\right]^{p_{k}} \geq \frac{\varepsilon}{2}\right\} .
\end{gathered}
$$

Since both the sets on the right hand of the relation (3.1) are belong to $I$ so the set on the left hand side of the inclusion relation belongs to $I$. This completes the proof of the theorem.

Theorem

$w_{0}^{I}\left[A, \Delta^{s}, M_{1}, p,\|., \ldots \ldots\|,\right] \cap w_{0}^{I}\left[A, \Delta^{s}, M_{2}, p,\|, \ldots \ldots .\|.\right] \subseteq w_{0}^{I}\left[A, \Delta^{s}, M_{1},+M_{2}, p,\|., \ldots \ldots\|,\right]$.

Proof. Let $x=\left(x_{k}\right) \in w_{0}^{I}\left[A, \Delta^{s}, M_{1}, p,\|., \ldots . .\|,\right] \cap w_{0}^{I}\left[A, \Delta^{s}, M_{2}, p,\|., \ldots . .\|,\right]$.

Then by the following inequality the result follows

$$
\begin{gathered}
\sum_{k=1}^{\infty} a_{n k}\left[\left(M_{1}+M_{2}\right)\left(\left\|\frac{\Delta^{s} x_{k}}{\rho}, z_{1}, z_{2}, \ldots, z_{n-1}\right\|\right)\right]^{p_{k}} \\
\leq D \sum_{k=1}^{\infty} a_{n k}\left[M_{1}\left(\left\|\frac{\Delta^{s} x_{k}}{\rho}, z_{1}, z_{2}, \ldots, z_{n-1}\right\|\right)\right]^{p_{k}}+D \sum_{k=1}^{\infty} a_{n k}\left[M_{2}\left(\left\|\frac{\Delta^{s} x_{k}}{\rho}, z_{1}, z_{2}, \ldots, z_{n-1}\right\|\right)\right]^{p_{k}} .
\end{gathered}
$$


Hence

$$
\begin{aligned}
& \left\{n \in \mathbf{N}: \sum_{k=1}^{\infty} a_{n k}\left[\left(M_{1}+M_{2}\right)\left(\left\|\frac{\Delta^{s} x_{k}}{\rho}, z_{1}, z_{2}, \ldots, z_{n-1}\right\|\right)\right]^{p_{k}} \geq \varepsilon\right\} \\
& \subseteq\left\{n \in \mathbf{N}: D \sum_{k=1}^{\infty} a_{n k}\left[M_{1}\left(\left\|\frac{\Delta^{s} x_{k}}{\rho}, z_{1}, z_{2}, \ldots, z_{n-1}\right\|\right)\right]^{p_{k}} \geq \frac{\varepsilon}{2}\right\} \\
& \cup\left\{n \in \mathbf{N}: D \sum_{k=1}^{\infty} a_{n k}\left[M_{2}\left(\left\|\frac{\Delta^{s} x_{k}}{\rho}, z_{1}, z_{2}, \ldots, z_{n-1}\right\|\right)\right]^{p_{k}} \geq \frac{\varepsilon}{2}\right\} .
\end{aligned}
$$

Since both the sets on the right hand are belong to $I$ so the set on the left hand side of the inclusion relation belongs to $I$. This completes the proof of the theorem.

Theorem 3.5. The inclusions $X\left[\Delta^{s-1}, M, p,\|. \ldots \ldots\|,\right] \subseteq X\left[A, \Delta^{s}, M, p,\|. \ldots \ldots\|,\right]$, are strict for $s \geq 1$. In general $X\left[\Delta^{j}, M, p,\|., \ldots .\|.\right] \subseteq X\left[A, \Delta^{s}, M, p,\|, \ldots . .\|,\right]$, for $j=$ $0,1,2, s^{-1}$ and the inclusions are strict, where $X=w_{0}^{I}, w^{I}$ and $w_{\infty}^{I}$.

Proof. We give the proof for $w_{0}^{I}\left[A, \Delta^{s-1}, M, p,\|. \ldots \ldots\|,\right]$ only. The others can be proved by similar argument. Let $x=\left(x_{k}\right)$ be any element in the space $w_{0}^{I}\left[A, \Delta^{s-1}, M, p,\|. \ldots \ldots\|,\right]$. Let $\varepsilon>0$ be given. Then there exists $\rho>0$ such that the set

$$
\left\{n \in \mathbf{N}: \sum_{k=1}^{\infty} a_{n k}\left[M\left(\left\|\frac{\Delta^{s-1} x_{k}}{\rho}, z_{1}, z_{2}, \ldots, z_{n-1}\right\|\right)\right]^{p_{k}} \geq \varepsilon\right\} \in I .
$$

Since $M$ is non-decreasing and convex, it follows that

$$
\begin{aligned}
& \sum_{k=1}^{\infty} a_{n k}\left[M\left(\left\|\frac{\Delta^{s} x_{k}}{2 \rho}, z_{1}, z_{2}, \ldots, z_{n-1}\right\|\right)\right]^{p_{k}}=\sum_{k=1}^{\infty} a_{n k}\left[M\left(\left\|\frac{\Delta^{s-1} x_{k+1}-\Delta^{s-1} x_{k}}{2 \rho}, z_{1}, z_{2}, \ldots, z_{n-1}\right\|\right)\right]^{p_{k}} \\
& \leq D \sum_{k=1}^{\infty} a_{n k}\left[\frac{1}{2} M\left(\left\|\frac{\Delta^{s-1} x_{k+1}}{\rho}, z_{1}, z_{2}, \ldots, z_{n-1}\right\|\right)\right]^{p_{k}}+D \sum_{k=1}^{\infty} a_{n k}\left[\frac{1}{2} M\left(\left\|\frac{\Delta^{s-1} x_{k}}{\rho}, z_{1}, z_{2}, \ldots, z_{n-1}\right\|\right)\right]^{p_{k}} \\
& \leq D H \sum_{k=1}^{\infty} a_{n k}\left[M\left(\left\|\frac{\Delta^{s-1} x_{k+1}}{\rho}, z_{1}, z_{2}, \ldots, z_{n-1}\right\|\right)\right]^{p_{k}}+D H \sum_{k=1}^{\infty} a_{n k}\left[M\left(\left\|\frac{\Delta^{s-1} x_{k}}{\rho}, z_{1}, z_{2}, \ldots, z_{n-1}\right\|\right)\right]^{p_{k}},
\end{aligned}
$$

where $H=\max \left\{1,\left(\frac{1}{2}\right)^{G}\right\}$.

Thus we have

$$
\begin{aligned}
& \left\{n \in \mathrm{N}: \sum_{k=1}^{\infty} a_{n k}\left[M\left(\left\|\frac{\Delta^{s} x_{k}}{2 \rho}, z_{1}, z_{2}, \ldots, z_{n-1}\right\|\right)\right]^{p_{k}} \geq \varepsilon\right\} \\
& \subseteq\left\{n \in \mathrm{N}: D H \sum_{k=1}^{\infty} a_{n k}\left[M\left(\left\|\frac{\Delta^{s-1} x_{k+1}}{\rho}, z_{1}, z_{2}, \ldots, z_{n-1}\right\|\right)\right]^{p_{k}} \geq \frac{\varepsilon}{2}\right\} \\
& \cup\left\{n \in \mathrm{N}: D H \sum_{k=1}^{\infty} a_{n k}\left[M\left(\left\|\frac{\Delta^{s-1} x_{k}}{\rho}, z_{1}, z_{2}, \ldots, z_{n-1}\right\|\right)\right]^{p_{k}} \geq \frac{\varepsilon}{2}\right\}
\end{aligned}
$$

Since both the sets in the right side of the relation (3.2) belongs to $I$, therefore we get the set

$$
\left\{n \in \mathbf{N}: \sum_{k=1}^{\infty} a_{n k}\left[M\left(\left\|\frac{\Delta^{s} x_{k}}{2 \rho}, z_{1}, z_{2}, \ldots, z_{n-1}\right\|\right)\right]^{p_{k}} \geq \varepsilon\right\} \in I .
$$

The inclusion is strict follows from the following example. 
Example 3.1. Let $M(x)=x$, for all $x \in[0, \infty), p_{k}=1$, for all $k \in \mathbf{N}$ and $A=(C, 1)$, i. e., the Cesàro matrix, Consider a sequence $x=\left(x_{k}\right)=\left(k^{s}\right)$. Then $x=\left(x_{k}\right)$ belongs to $w_{0}^{I}\left[\Delta^{s}, M, p,\|., \ldots . .\|,\right]$ but does not belong to $w_{0}^{I}\left[\Delta^{s-1}, M, p,\|., \ldots .\|.\right]$, because $\Delta^{s}$ $x_{k}=0$ and $\Delta^{s-1} x_{k}=(-1)^{s-1}(s-1)$ !.

Theorem 3.6. For any two sequences $p=\left(p_{k}\right)$ and $q=\left(q_{k}\right)$ of positive real numbers and for any two n-norms $\| .$, ., ..., . $\|_{1}$ and $\|., ., . . ., .\|_{2}$ on $X$, then the following holds: $X\left[A, \Delta^{s}, M, p,\|., \ldots \ldots\|_{1}\right] \cap X\left[A, \Delta^{s}, M, q,\|., \ldots .\|_{2}\right] \neq \emptyset$, where $X=w_{0}^{I}$, w and $w_{\infty}^{I}$.

Proof. Since the zero element belongs to each of the above classes of sequences, thus the intersection is nonempty.

Theorem 3.7. The sequence spaces $w_{0}^{I}\left[A, \Delta^{s}, M, p,\|, \ldots \ldots,\|.\right]$ and $w_{\infty}^{I}\left[A, \Delta^{s}, M, p,\|., \ldots . .\|,\right]$ are normal as well as monotone.

Proof. We give the proof for $w_{0}^{I}\left[A, \Delta^{s}, M, p,\|, \ldots . .\|,\right]$ only. Let $x=\left(x_{k}\right) \in$ $w_{0}^{I}\left[A, \Delta^{s}, M, p,\|., \ldots . .\|.\right]$ and $\alpha=\left(\alpha_{k}\right)$ be a sequence of scalars such that $\left|\alpha_{k}\right| \leq 1$ for all $k \in \mathbf{N}$. Then for given $\varepsilon>0$ we have

$$
\begin{aligned}
& \left\{n \in \mathrm{N}: \sum_{k=1}^{\infty} a_{n k}\left[M\left(\left\|\frac{\Delta^{s}\left(\alpha_{k} x_{k}\right)}{\rho}, z_{1}, z_{2}, \ldots, z_{n-1}\right\|\right)\right]^{p_{k}} \geq \varepsilon\right\} \\
\subseteq & \left\{n \in \mathrm{N}: E \sum_{k=1}^{\infty} a_{n k}\left[M\left(\left\|\frac{\Delta^{s} x_{k}}{\rho}, z_{1}, z_{2}, \ldots, z_{n-1}\right\|\right)\right]^{p_{k}} \geq \varepsilon\right\} \in I,
\end{aligned}
$$

where $E=\max \left\{1,\left|\alpha_{k}\right|^{G}\right\}$.

Hence $\left(\alpha_{k} x_{k}\right) \in w_{0}^{I}\left[A, \Delta^{s}, M, p,\|., \ldots, \ldots\|.\right]$. Thus the space $w_{0}^{I}\left[A, \Delta^{s}, M, p,\|, \ldots . .,\|.\right]$ is normal. Also from the Lemma 2.1, it follows that $w_{0}^{I}\left[A, \Delta^{s}, M, p,\|., \ldots \ldots,\|.\right]$ is monotone. This completes the proof of the theorem.

\section{Acknowledgements}

The author would like to thank the referees for their careful reading of the manuscript and for their helpful suggestions

\section{Competing interests}

The authors declare that they have no competing interests.

Received: 20 November 2011 Accepted: 16 April 2012 Published: 16 April 2012

\section{References}

1. Zygmund, A: Trigonometric Series. pp. 233-239. Cambridge University Press, Cambridge, UK51(2): (2011)

. Fast, H: Sur la convergence statistique. Collog Math. 2, 241-244 (1951)

3. Schoenberg, IJ: The integrability of certain functions and related summability methods. Am Math Month. 66, 361-375 (1959)

4. Fridy, JA: On statistical convergence. Analysis. 5, 301-313 (1985)

5. Kostyrko, P, Salat, T, Wilczyski, W: On I-convergence, real analysis exchange. 26(2):669-686 (2000-2001)

6. Savas, E: $\Delta^{m}$-strongly summable sequence spaces in 2-normed spaces defined by ideal convergence and an Orlicz function. Appl Math Comput. 217, 271-276 (2010)

7. Savas, E: A-sequence spaces in 2-normed space defined by ideal convergence and an Orlicz function. Abst Appl Anal 2011(1-9):1-9 (2011). Article ID 741382

8. Tripathy, BC, Hazarika, B: Paranorm I-convergent sequence spaces. Math Slovaca. 59(4):485-494 (2009)

9. Tripathy, BC, Hazarika, B: Some I-convergent sequence spaces defined by Orlicz functions. Acta Math Appl Sinica. 27(1):149-154 (2011)

10. Kizmaz, H: On certain sequence spaces. Canad Math Bull. 24(2):169-176 (1981)

11. Et, M, Colak, R: On generalized difference sequence spaces. Soochow J Math. 21(4):147-169 (1985)

12. Gahler, S: Linear 2-normietre Raume. Math Nachr. 28, 1-43 (1965)

13. Misiak, A: $n$-inner product spaces. Math Nachr. 140, 299-329 (1989)

14. Gunawan, $\mathrm{H}$ : The spaces of $p$-summable sequences and its natural n-norm. Bull Aust Math Soc. 64, 137-147 (2001)

15. Gunawan, H, Mashadi, M: On n-normed spaces. Int J Math Math Sci. 27(10):631-639 (2001)

16. Gunawan, H, Mashadi, M: On finite dimensional 2-normed spaces. Soochow J Math. 27(3):147-169 (2001)

17. Gurdal, M: On ideal convergent sequences in 2-normed spaces. Thai J Math. 4(1):85-91 (2006) 
18. Sahiner, A, Gurdal, M, Soltan, S, Gunawan, H: Ideal convergence in 2-normed spaces. J Math. 11, 1477-1484 (2007)

19. Savas, E: On some new sequence spaces in 2-normed spaces using ideal convergence and an Orlicz function. J Ineq Appl 1-9 (2010). Article ID: 482392, doi:10.1155/2010/482392

20. Gurdal, M, Sahiner, A: Ideal convergence in $n$-normal spaces and some new sequence spaces via $n$-norm. J Funda Sci. 4, 233-244 (2008)

21. Hazarika, B: On paranormed Ideal convergent generalized difference strongly summable sequence spaces defined over n-normed spaces. ISRN Math Anal 2011, 17 (2011). Article ID 317423

22. Sava, E: Some new double sequence spaces defined by Orlicz function in nn-normed space. J Inequal Appl. 1-9 (2011)

23. Krasnoselskii, MA, Rutickii, YB: Convex functions and Orlicz spaces. Groningen, Netherlands (1961)

24. Lindenstrauss, J, Tzafriri, L: On Orlicz sequence spaces. Israel J Math. 10, 379-390 (1971)

25. Parashar, SD, Choudhary, B: Sequence spaces defined by Orlicz functions. Indian J Pure Appl Math. 25(4):419-428 (1994)

26. Mursaleen, M, Khan, MA: Qamaruddin, difference sequence spaces defined by Orlicz functions. Demonstratio Math. 32, $145-150$ (1999)

27. Tripathy, BC, Altin, Y, Et, M: Generalized difference sequence spaces on seminormed spaces defined by Orlicz function. Math Slovaca. 58(3):315-324 (2008)

28. Tripathy, BC, Dutta, $\mathrm{H}$ : On some new paranormed difference sequence spaces defined by Orlicz functions. Kyungpook Math J. 50(1):59-69 (2010)

29. Sahiner, A, Gurdal, M: New sequence spaces in $n$-normed spaces with respect to an Orlicz function. Alligarh Bull Math. 27, 53-58 (2008)

30. Kamthan, PK, Gupta, M: Sequence spaces and series. Marcel Dekkar, New York (1980)

doi:10.1186/1029-242X-2012-87

Cite this article as: Savas: On generalized $A$ - difference strongly summable sequence spaces defined by ideal

convergence on a real $n$-normed space. Journal of Inequalities and Applications 2012 2012:87.

\section{Submit your manuscript to a SpringerOpen ${ }^{\odot}$} journal and benefit from:

- Convenient online submission

- Rigorous peer review

- Immediate publication on acceptance

- Open access: articles freely available online

- High visibility within the field

- Retaining the copyright to your article

Submit your next manuscript at $\boldsymbol{\nabla}$ springeropen.com 